\title{
The Relationship Between Infarct-Related Arteries Patency with C-Reactive Protein/Albumin Ratio Before Primary Percutaneous Coronary Intervention in Patients with ST-Segment Elevation Myocardial Infarction
}

\author{
Halil Akın${ }^{1}$, Önder Bilge ${ }^{2}$, Bernas Altintas ${ }^{2}$, Rojhat Altındağ ${ }^{2}$, Hüseyin Ede ${ }^{3}$, and Mehmet \\ Sahin Adiyaman ${ }^{2}$ \\ ${ }^{1}$ Sinop Ataturk Devlet Hastanesi \\ ${ }^{2}$ Diyarbakır Gazi Yaşargil Eğitim ve Araştırma Hastanesi \\ ${ }^{3}$ Hamad General Hospital
}

April 20, 2021

\begin{abstract}
Background: Inflammatory markers such as C-reactive protein and Albumin have previously been associated with poor prognosis in ST-elevation myocardial infarction (STEMI). Objective: The present study aims to investigate the relationship between the infarct-related arteries (IRA) patency and C-reactive protein/Albumin ratio (CAR) before primary percutaneous intervention (p-PCI) in patients with STEMI. Methods A total of 822 patients who underwent primary PCI (p-PCI) for acute STEMI were included in this study. Patients were divided into two groups according to IRA patency as TIMI flow 0-1 (n $=551)$ and TIMI flow 2-3 $(\mathrm{n}=271)$. CAR ratio measured at admission was compared with IRA patency. Results: The average age of 822 patients was $55 \pm 12$, and $84.3 \%$ (693) of the patients were male. The mean CAR level of the patients was determined as 0.26 (0.08-0.48). CAR level was statistically significantly higher in TIMI flow 0-1 group when compared to TIMI flow 2-3 group [0.31 $(0.09-0.51)$ vs $0.23(0.06-0.42) ; \mathrm{p}<0.001]$. In the multivariate logistic regression analysis a significant relation was found between CAR (odds ratio [OR]:1.56, 95\% confidence interval [CI]:1.22-1.97, $\mathrm{p}<0.001$ ), and neutrophil count (OR:1.72, 95\% CI:1.33-2.25, $\mathrm{p}<0.001$ ) in patients with TIMI flow 0-1 Conclusion: An inflammation-based risk index, CAR measured at admission in patients with anterior STEMI has been found to be a useful prognostic tool for predicting adverse cardiovascular outcomes. However, this finding needs to be confirmed in future prospective studies.
\end{abstract}

\section{Introduction}

Although a decrease is seen in the mortality rate with the widespread of primary percutaneous coronary intervention (p-PCI) in patients with ST-elevation myocardial infarction (STEMI), there is still a high rate of mortality ${ }^{1}$. The rapid and successful revascularization of the infarct-related artery (IRA) has proven to be the most effective treatment option in patients with STEMI and improves clinical outcomes. IRA patency before p-PCI cause the short duration of ischemia in STEMI patients, thus decreasing complications such as infarction-related heart failure, cardiogenic shock and mortality, and high procedure success ${ }^{2}$.

The use of inflammatory biomarkers has been increasing in the diagnosis and screening of atherosclerotic heart disease (AHD) $)^{3}$. Among the most frequently used biomarkers for this purpose, C-reactive protein $(\mathrm{CRP})$ and albumin are known to have a strong association with individual AHD formation and presentation forms. ${ }^{4,5} \mathrm{C}$ reactive protein/Albumin ratio (CAR) reflects the stability of albumin and CRP levels in the body. It has 
been shown to reflect the inflammatory status and prognosis better than the hs-CRP or albumin alone in critical patients, acute medical conditions, and cancer patients ${ }^{6-9}$.

Detection of IRA patency in p-PCI in patients with STEMI provides predicting mortality and morbidity rates of the patients ${ }^{10}$. Therefore, in the present study, it was aimed to investigate the relationship between IRA patency before p-PCI and CAR in patients with STEMI.

\section{Methods}

\section{Study Population}

Patients with STEMI who underwent Primary PCI (p-PCI) in our hospital between February 2014 and December 2017 were included in this retrospective study. Inclusion criteria were as follows: onset of symptoms $<6$ hours before p-PCI; ST-segment elevation $>0.2 \mathrm{mV}$ in 2 or more contiguous precordial or extremity leads. Left and right coronary angiograms were obtained before the attempted angioplasty. Exclusion criteria were venous graft-related infarcts, non-gradable IRA flow due to technical reasons, concurrent pericardial disease, chronic pulmonary disease, pulmonary hypertension, valvular heart disease (moderate to severe insufficiency and/or stenosis), acute pulmonary embolism, history of cardiac arrest before admission Patients with a history of the previous CAD treated with PCI or coronary artery bypass grafting, malignancy, active infection, and connective tissue disorder were excluded from the study. The patients were divided into two as TIMI 0-1 and TIMI 2-3 according to the IRA patency rate. Of the patients included in this study, $67 \%$ were of TIMI flow 0-1 $(\mathrm{n}=551), 33 \%$ of TIMI flow 2-3 $(\mathrm{n}=271)$. Informed consent of each subject's approval of the Local Ethics Committee with the principles of the Helsinki Declaration was obtained.

\section{Coronary Angiography}

Coronary angiography (CAG) was performed within 90 minutes of hospital admission. All patients received dual antiplatelet therapy with aspirin $(300 \mathrm{mg})$ and clopidogrel $(600 \mathrm{mg})$ or ticagrelor $(180 \mathrm{mg})$ loading dose before CAG. Preprocedural anticoagulation consisted of intravenous unfractionated heparin $(70 \mathrm{IU} / \mathrm{kg})$ in all cases. Coronary angiograms were recorded to digital media for quantitative analysis (DICOM viewer, MedCom GmbH, Darmstadt, Germany). Digital angiograms were evaluated by 2 experienced cardiologists who were blinded to other patient information. In case of any conflicts regarding the assessments, an agreement was reached by consensus. The degree of coronary flow before PCI was classified by thrombolysis in myocardial infarction (TIMI) grade flow as assessed by the investigators. Patients with TIMI grade 2 or 3 flow in the IRA were considered to have a patent vessel. Primary PCI with stent implantation was performed according to current guidelines. The purpose of the p-PCI procedure was to obtain residual stenosis of $<20 \%$ in IRA by visual evaluation. An optimal angiographic result was defined as the presence of TIMI grade 3 flow in the IRA following p-PCI. An unsuccessful procedure was defined as a procedure resulting in TIMI grade $0-1$. Use of glycoprotein IIb/IIIa inhibitors (i.e. tirofiban) was left to the discretion of the attending physician. Complete ST-segment resolution was defined as a reduction of $>70 \%$ in the summed 12-lead extent of ST-segment elevation from baseline to the post-procedural electrocardiogram, which was recorded at 90th minute after the first balloon inflation.

\section{Laboratory Measurements}

Routine complete blood cell count and blood evaluations for determining the blood glucose, creatinine, albumin, CRP, and troponin I levels were conducted using the admission blood samples. Troponin I levels was measured every 6 hours until they peaked. The albumin and CRP levels were measured using a Roche Diagnostics Cobas 8000 c502 analyzer ((Indianapolis, USA). The CAR was calculated as the ratio of CRP to the albumin level multiplied by 100. The estimated glomerular filtration rate (eGFR) was calculated using the Cockcroft- Gault equation. Left ventricular ejection fraction (LVEF) was measured using the modified Simpson method.

\section{Statistical Analyses}

Data were analyzed using the SPSS 24.0 version (SPSS Inc, Chicago, Illinois). Normality of the data was 
determined using the Kolmogorov-Smirnov test. The continuous variables with a normal distribution are presented as mean + standard deviation (SD) values, while those without a normal distribution are presented as median and interquartile range values. Frequency distribution was calculated for the categorical variables (numbers and percentages). The continuous variables of the 2 groups were compared using the Student t-test or the Mann-Whitney U test. Categorical data were compared using the chi-square test or the Fisher exact test. Multivariate logistic regression analysis was used to determine independent predictors of coronary patency using variables found to be significant in univariate analysis $(\mathrm{P}<0.05)$. Receiver-operating characteristic (ROC) analyses were used to compare the performance power of the CAR, CRP, and albumin for coronary patency. The predictive validities were quantified as the area under the ROC curves (c statistics), and the comparisons of statistics were performed by De long's test.

\section{Results}

A total of 822 patients who underwent p-PCI due to STEMI and have IRA patency rates were included in the study. The average age of 822 patients was $55 \pm 12$, and $84.3 \%$ (693) of the patients were male. The mean CAR level of all study patients was $0.26(0.08-0.48)$. The patients were divided into two as TIMI $0-1$ and TIMI 2-3 according to the IRA patency rate. Of the patients included in this study, $67 \%$ were of TIMI flow $0-1(\mathrm{n}=551), 33 \%$ of TIMI flow $2-3(\mathrm{n}=271)$.

The baseline demographic, clinical, laboratory and angiographic characteristic features of the study patients according to TIMI flow are listed in Table 1 . CAR level was found statistically significantly higher In the TIMI flow 0-1 group compared to TIMI flow 2-3 group [0.31 (0.09-0.51) vs $0.23(0.06-0.42) ; \mathrm{p}<0.001]$. In the TIMI flow 0-1 group, there were statistically significant decreases in hemoglobin, platelet count, albumin level, e-GFR, and left ventricular EF, while there were significant increases in neutrophil count, C-reactive protein, lymphocyte count and heart rate when compared to TIMI 2-3 group (Table 1).

Table 1. Baseline Demographic, Clinical, Laboratory and Angiographic characteristic of study patients

Baseline IRA patency

\begin{tabular}{|c|c|c|c|c|}
\hline & $\begin{array}{l}\text { All patients } \\
(\mathrm{n}=822)\end{array}$ & TIMI $0-1 \quad(\mathrm{n}=551)$ & TIMI 2-3 $(\mathrm{n}=271)$ & $\mathrm{p}$ value \\
\hline Age [year] & $55 \pm 12$ & $54 \pm 11$ & $56 \pm 12$ & 0.76 \\
\hline $\begin{array}{l}\text { Male gender [n } \\
(\%)]\end{array}$ & $693(84.3)$ & $466(84.6)$ & $227(83.8)$ & 0.47 \\
\hline $\begin{array}{l}\text { Diabetes mellitus } \\
{[\mathrm{n}(\%)]}\end{array}$ & $179(21.8)$ & $124(22.5)$ & $55(20.3)$ & 0.06 \\
\hline $\begin{array}{l}\text { Hypertension [n } \\
(\%)]\end{array}$ & $320(38.9)$ & $202(36.7)$ & $118(43.5)$ & 0.31 \\
\hline $\begin{array}{l}\text { Hyperlipidemia [n } \\
(\%)]\end{array}$ & $234(28.4)$ & $153(27.7)$ & $80(29.5)$ & 0.55 \\
\hline Smoking [n (\%)] & $295(35.8)$ & $209(37.9)$ & $86(31.7)$ & 0.25 \\
\hline $\begin{array}{l}\text { Family history of } \\
\text { CAD }[\mathrm{n}(\%)]\end{array}$ & $186(22.6)$ & $128(23.2)$ & $58(21.6)$ & 0.11 \\
\hline $\begin{array}{l}\text { KILLIP class }>1 \\
\text { on admission }\end{array}$ & $103(12.5)$ & $78(14.2)$ & $25(9.2)$ & 0.30 \\
\hline $\begin{array}{l}\text { Systolic blood } \\
\text { pressure [mmHg] }\end{array}$ & $135 \pm 21$ & $136 \pm 22$ & $133 \pm 20$ & 0.43 \\
\hline Heart rate $[/ \mathrm{min}]$ & $86 \pm 18$ & $92 \pm 16$ & $76 \pm 15$ & 0.02 \\
\hline Hemoglobin [g/dl] & $12.9 \pm 1.7$ & $12.1 \pm 1.8$ & $14.0 \pm 1.5$ & 0.01 \\
\hline $\begin{array}{l}\text { White blood cell } \\
\text { count }\left[10^{3} / \mu \mathrm{l}\right]\end{array}$ & $12.0 \pm 3.3$ & $12.4 \pm 3.4$ & $11.2 \pm 3.0$ & 0.74 \\
\hline $\begin{array}{l}\text { Platelet count } \\
{\left[10^{5} / \mu \mathrm{l}\right]}\end{array}$ & $259 \pm 68$ & $255 \pm 74$ & $271 \pm 58$ & $<0.001$ \\
\hline
\end{tabular}




\begin{tabular}{|c|c|c|c|c|}
\hline $\begin{array}{l}\text { Neutrophil count } \\
{\left[10^{3} / \mu l\right]}\end{array}$ & $9.2 \pm 3.2$ & $9.7 \pm 3.3$ & $8.2 \pm 2.8$ & $<0.001$ \\
\hline $\begin{array}{l}\text { Lymphocyte } \\
\text { count }\left[10^{3} / \mu \mathrm{l}\right]\end{array}$ & $1.8 \pm 1.3$ & $1.9 \pm 1.5$ & $1.7 \pm 1.2$ & $<0.001$ \\
\hline $\begin{array}{l}\text { Neutrphil to } \\
\text { Lymphocyte ratio }\end{array}$ & $4.9(3.2-7.4)$ & $5.1(3.6-8.2)$ & $4.7(2.9-6.1)$ & 0.13 \\
\hline $\begin{array}{l}\text { Fasting blood } \\
\text { glucose }[\mathrm{mg} / \mathrm{dl}]\end{array}$ & $148.2 \pm 72.2$ & $151.2 \pm 76.3$ & $142.2 \pm 62.5$ & 0.27 \\
\hline $\begin{array}{l}\text { Estimated GFR } \\
{[\mathrm{mL} / \mathrm{min}]}\end{array}$ & $91.6 \pm 23.5$ & $90.8 \pm 24.2$ & $93.5 \pm 25.1$ & $<0.001$ \\
\hline $\begin{array}{l}\text { C-reactive protein } \\
{[\mathrm{mg} / \mathrm{dl}]}\end{array}$ & $0.86(0.51-1.36)$ & $0.92(0.59-1.55)$ & $0.76(0.45-1.36)$ & $<0.001$ \\
\hline Albumin [g/dl] & $3.8(3.6-4.1)$ & $3.7(3.5-4.0)$ & $3.9(3.6-4.1)$ & $<0.001$ \\
\hline CAR [ x100] & $22(8-46)$ & $26(9-49)$ & $17(6-42)$ & $<0.001$ \\
\hline LVEF [\%] & $48.5 \pm 7.2$ & $47.3 \pm 8.1$ & $50.9 \pm 6.7$ & 0.01 \\
\hline $\begin{array}{l}\text { Total ischemia } \\
\text { time (min) }\end{array}$ & $169(108-245)$ & $176(116-255)$ & $145(92-228)$ & 0.32 \\
\hline IRA [n (\%)] & & & & 0,62 \\
\hline $\operatorname{LAD}[\mathrm{n}(\%)]$ & $419(51.0)$ & $275(50.0)$ & $144(53.1)$ & \\
\hline $\operatorname{LCX}[\mathrm{n}(\%)]$ & $110(13.4)$ & $74(13.4)$ & $36(13.3)$ & \\
\hline $\mathrm{RCA}[\mathrm{n}(\%)]$ & $282(34.3)$ & $193(35.0)$ & $89(32.8)$ & \\
\hline $\begin{array}{l}\text { Other coronary } \\
\text { arteries }[\mathrm{n}(\%)]\end{array}$ & $11(1.3)$ & $9(1.6)$ & $2(0.7)$ & \\
\hline $\begin{array}{l}\text { Number of } \\
\text { diseased vessel[n } \\
(\%)]\end{array}$ & & & & 0.12 \\
\hline 1 vessel [n (\%)] & $513(62.4)$ & $334(60.6)$ & $179(66.1)$ & \\
\hline $2-3$ vessel $[\mathrm{n}(\%)]$ & $309(37.6)$ & $217(39,4)$ & $70(33.9$ & \\
\hline
\end{tabular}

Data are expressed as mean \pm SD for normaly distributed data or count (percentage) for categorical variables; CAD, Coronary artery disease; CAR, C-reactive protein to albumin ratio; eGFR, estimated glomerular filtration rate; IRA, Infarct releated artery; LAD, Left anterior descending artery; LVEF, left ventricular ejection fraction; LCX, Left circumflex artery; RCA, right coronary artery; TIMI, Thrombolysis in myocardial infarction

The univariate logistic regression analysis revealed that the CAR, hemoglobin, platelet count, albumin level, eGFR, left ventricular EF, neutrophil count, lymphocyte count, and heart rate values were associated with the IRA patency. CRP was not included in the analysis because it showed multicollinearity with CAR. In the multivariate logistic regression analysis to determine the relationship with IRA patency in patients with STEMI, the CAR (odds ratio [OR]:1.56, 95\% confidence interval [CI]:1.22-1.97, $\mathrm{p}<0.001$ ) and neutrophil count (OR:1.72, 95\% CI:1.33-2.25, $\mathrm{p}<0.001$ ) were found to be associated with TIMI 0-1 flow (Table 2).

Table 2 . Univariate and multivariate logistic regression analysis for baseline IRA patency (TIMI 0/1)

\begin{tabular}{lllllll}
\hline Variable & Univarite & Univarite & Univarite & Multivariate & Multivariate & Multivariate \\
\hline & Unadjusted OR & $95 \%$ CI & pvalue & Adjusted OR & $95 \%$ CI & pvalue \\
Albumin & 1.45 & $0.95-1.57$ & 0.05 & & & \\
eGFR & 0.94 & $0.74-1.03$ & 0.04 & & & \\
Lymphocyte & 1.25 & $1.08-1.45$ & 0.02 & & & \\
Neutrophil & 1.83 & $1.28-2.34$ & $<0.001$ & 1.72 & $1.33-2.25$ & $<0.001$ \\
Platelet & 1.49 & $0.91-1.78$ & 0.08 & & &
\end{tabular}




\begin{tabular}{lllllll}
\hline Variable & Univarite & Univarite & Univarite & Multivariate & Multivariate & Multivariate \\
\hline Hemoglobin & 1.38 & $0.89-1.42$ & 0.12 & & & \\
Heart rate & 1.07 & $0.92-1.21$ & 0.74 & & & \\
CAR & 1.80 & $1.21-2.41$ & $<0.001$ & 1.56 & $1.22-1.97$ & $<0.001$ \\
\hline
\end{tabular}

$\mathrm{Cl}=$ confidence interval; $\mathrm{OR}=$ oddsratio; $\mathrm{CAD}$, Coronary artery disease; CAR, C-reactive protein to albumin ratio; eGFR, estimated glomerular filtration rate; IRA, Infarct releated artery

In the ROC analysis performed to predict TIMI flow 0-1, the optimal predictive value for CAR was 0.375 with $48 \%$ sensitivity and $84 \%$ specificity. The ROC curves were compared to evaluate whether CAR has additional distinctive value on the CRP and albumin levels. CAR's Area Under Curve (AUC) value was statistically significantly higher compared to CRP (AUC: $0.59 ; 95 \% \mathrm{CI}$ : $0.55-0.63, \mathrm{p}=0.014$ ) and albumin (AUC: $0.57 ; 95 \%$ CI: $0.53-0.62, \mathrm{p}=0.028$ ) in predicting TIMI flow 0-1 (Figure 1).

Figure 1. The receiver operating characteristic (ROC) curve comparison of C-reactive protein (CRP), albumin, and C-reactive protein to albumin ratio (CAR) in the prediction of baseline IRA patency

\section{Hosted file}

image1.wmf available at https://authorea.com/users/408942/articles/518759-the-relationshipbetween-infarct-related-arteries-patency-with-c-reactive-protein-albumin-ratio-beforeprimary-percutaneous-coronary-intervention-in-patients-with-st-segment-elevationmyocardial-infarction

\section{Discussion}

To the best of our knowledge, this is the first study evaluating the relationship between the ratio of CAR with patency of IRA in patients with STEMI before the p-PCI. It revealed that CAR might be related to the IRA patency before p-PCI $(\mathrm{p}<0.001)$.

p-PCI is the gold standard treatment method in patients with STEMI. However, the duration of infarction is the most important factor affecting the clinical results of the patients ${ }^{11}$. In the HORIZON-AMI study, early IRA patency in patients with STEMI undergoing primary PCI is associated with better TIMI flow and myocardial blush post PCI and is an independent predictor of lower one-year mortality ${ }^{12}$. Christopoulos $\mathrm{C}$ et al. associated the IRA patency in patients with STEMI with increased endogenous thrombolysis, decreased platelet activity, and resulting decreased ischemia time ${ }^{13}$. Shortness of ischemia duration, decreased complications such as heart failure due to infarction, malignant arrhythmia, cardiogenic shock, mortality, and high procedure success in patients with STEMI ${ }^{2}$. Our study, in accordance with other studies, with statistical significance in the TIMI 0-1 group compared to the TIMI 2-3 group; There was a decrease in eGFR and left ventricular $\mathrm{EF}$, and an increase in heart rate, which was associated with poor cardiovascular results (Table $1)$.

Inflammation plays an important role in determining both pathogenesis and prognosis in STEMI ${ }^{14,15}$. Management of the occurring systemic inflammatory process affects the prognosis of the disease ${ }^{16}$. Macrophage activation, free radical release and proinflammatory cytokines secreted from inflammatory cells increase in acute myocardial infarction ${ }^{17}$. Inflammation in STEMI causes an increase not only in the responsible plaque region but also in all systemic circulation and other plaques ${ }^{18}$. Besides, thrombus load in IRA creates microvascular plugs and causes no-reflow to increase the ischemic process and thus increases myocardial inflammation. As a result, an increase in inflammatory cells is seen in STEMI as an indicator of plaque inflammation as well as myocardial tissue destruction in the coronary arteries ${ }^{19}$. In the study by Pietila et al., it was found that the height of inflammatory markers showed the amount of destroyed myocardial tissue in patients who could not achieve IRA patency by giving thrombolytics ${ }^{20,21}$. In our study, consistent with previous studies, a decrease in the inflammatory marker albumin level and an increase in the number of neutrophils, C-reactive protein and lymphocytes were found (Table 1). Data investigating the relationship 
of IRA patency in patients with STEMI is limited. Doganay B. et al., who investigated IRA patency in patients with STEMI, have found a significant relationship between the co-peptit level and the IRA ${ }^{22}$, while Jing L. et al., however, found a significant relationship between the homocysteine level and IRA ${ }^{23}$. CAR, an inflammatory marker, reflects the stability of albumin and CRP levels within the body. Few studies have reported the relationship between ACS and CAR. In studies performed, high CAR elevation was associated with poor prognosis in patients with STEMI and stable angina pectoris ${ }^{24,25}$. In a study investigating the relationship between short-term major adverse cardiac events (MACE) and CAR, which included 652 ACS patients, it was seen that increased CAR increased the likelihood of developing $\mathrm{MACE}^{26}$. Another study has revealed that CAR could predict no-reflow in patients with ST-elevation myocardial infarction ${ }^{27}$. In our study, the value of CAR was found to be statistically higher in patients without IRA patency in patient with STEMI (Table 1).

In patients with STEMI, the IRA patency, which is well known for its effects on major cardiac side effects and mortality, results in a decreased duration of ischemia and necrotic area, and associated inflammation in the ischemic area ${ }^{28,29}$. However, this effect in the IRA patency is limited with the thrombus load. Because the increased thrombus load in the IRA patency will disrupt coronary perfusion after p-PCI with microvascular obstruction. Insufficient perfusion will lead to increased ischemic area and associated inflammation. Considering that using the parameters reflecting the inflammatory process in patients with ACS in determining thrombus load in the IRA patency will be useful for determining the prognosis of the patients, the relationship between IRA patency and CAR was investigated in patients with STEMI, and the rate of CAR was found to be significantly higher in patients with TIMI 0-1 flow in IRA $(\mathrm{p}<0.001)$.

\section{Conclusion}

CAR, an inflammatory marker in patients with STEMI, is known to influence the rates of mortality and morbidity. However, the relationship between CAR and IRA patency has not been investigated. This is the first study to reveal a significant relationship between the IRA patency and CAR before p-PCI in STEMI. The C-reactive protein/albumin ratio is an easy and reliable indicator that can be used to determine IRA patency in patients with STEMI.

\section{Limitations of the study}

The study has been carried out retrospectively. Methods such as cardiac nuclear imaging and cardiac MR, which are more specific and reliable, could be used to evaluate the reperfusion. However, their routine use is not possible in terms of cost and availability.

\section{Declaration of Conflicting Interests}

The author(s) declared no potential conflicts of interest with respect to the research, authorship, and/or publication of this article.

\section{Funding and Acknowledgements}

The author(s) received no financial support for the research, authorship, and/or publication of this article.

\section{REFERENCES}

1- Steg PG, James SK, Atar D, et al. ESC Guidelines for the management of acute myocardial infarction in patients presenting with ST-segment elevation: The Task Force on the management of ST-segment elevation ac te myocardial infarction of the European Society of Cardiology (ESC). Eur Heart J. 2012;33:2569-619.

${ }^{2}$-Zeymer U, Huber K, Fu Y, et al.; for the ASSENT-4 PCI Investigators. Impact of TIMI 3 patency before primary percutaneous coronary intervention for ST-elevation myocardial infarction on clinical outcome: Results from the ASSENT-4 PCI study. Eur Heart J Acute Cardiovasc Care 2012; 1: 136-142.

${ }^{3}$-Bisoendial RJ, Boekholdt SM, V rgeer M, et al. C-reactive protein is a mediator of cardiovascular disease. Eur Heart J. 2010;31:2087-91. 
4-Bisoendial RJ, Kast 1 in JJP, Peters SLM, et al. Effects of CRP infusion on endothelial function and coagulation in normocholesterolemic and hypercholesterolemic subjects. J Lipid Res. 2007;48:952-60.

${ }^{5}$-Oduncu V, Erkol A, Karabay CY, et al. The prognostic value of serum albumin levels on admission in patients with acute ST-segment elevation myocardial infarction undergoing a primary percutaneous coronary intervention. Coron Artery Dis. 2013;24:88-94

${ }^{6}$-Fairclough E, Cairns E, Hamilton J, et al. Evaluation of a modified early warning system for acute medical admissions and comparison with C-reactive protein/albumin ratio as a predictor of patient outcome. Clinical medicine. 2009;9(1):30-33.

7-Kinoshita A, Onoda H, Imai N, et al. The C-reactive protein/albumin ratio, a novel inflammation-based prognostic score, predicts outcomes in patients with hepatocellular carcinoma. Annals of surgical oncology. $2015 ; 22(3): 803-810$.

${ }^{8}$-Fairclough E, Cairns E, Hamil on J, et al. Evaluation of a modified early warning system for acute medical admissions and comparison with C-reactive protein/albumin ratio as a predictor of patient outcome. Clin Med (Lond). 2009;9:30-3.

${ }^{9}$-Kinoshita A, Onoda H, Imai N, et al. The C-reactive protein/albumin ratio, a novel inflammation-based prognostic score, predicts outcomes in patients with hepatocellular carcinoma. Ann SurgOncol.2015;22:80310.

10-Nallamothu BK, Normand SL, Wang Y, et al. Relation between door-to-balloon times and mortality after primary percutaneous coronary intervention over time: a retrospective study. Lancet 2015;385:1114-22.

11_ Zeymer U, Huber K, Fu Y, et al.; for the ASSENT-4 PCI Investigators. Impact of TIMI 3 patency before primary percutaneous coronary intervention for ST-elevation myocardial infarction on clinical outcome: Results from the ASSENT-4 PCI study. Eur Heart J Acute Cardiovasc Care 2012; 1: 136-142.

${ }^{12}$-Rakowski T, Dudek D, Dziewierz A, et al. Impact of infarct-related artery patency before primary PCI on outcome in patients with ST-segment elevation myocardial infarction: The HORIZONS-AMI trial. Eurointervention 2013; 8: 1307-1314.

${ }^{13}$ _Christopoulos C, Farag M, Sullivan K, et al. Impaired thrombolytic status predicts adverse cardiac events in patients undergoing primary percutaneous coronary intervention. Thromb. Haemost. 2017;117(3): $457-70$.

14- Stefanadis C, Diamantopoulos L, Vlachopoulos C, et al. Thermal heterogeneity within human atherosclerotic coronary arteries detected in vivo: a new method of detection by application of a special thermography catheter. Circulation 1999;99:1965-71.

15 - Fayad ZA, Fuster V, Fallon JT, et al. Noninvasive in vivo human coronary artery lumen and wall imaging using black-blood magnetic resonance imaging. Circulation 2000;102:506-10.

16 - Kottoor SJ, Arora RR. The utility of anti-inflammatory agents in cardiovascular disease: a novel perspective on the treatment of atherosclerosis. J Cardiovasc Pharmacol Ther. 2018-107424841877854

${ }^{17}$-Biasucci LM, D’Onofrio G, Liuzzo G, et al. Intracellular neutrophil myeloperoxidase is reduced in unstable angina and myocardial infarction, but its reduction is not related to ischemia. J Am Coll Cardiol 1996;27:6116 .

18-Buffon A, Biasucci LM, Liuzzo G, et al. Widespread coronary inflammation in unstable angina. N Engl J Med 2002;347: 5-12.

19-Barrett TO, Hennan JK, Marks RM, et al. C-reactive- protein-associated increase in myocardial infarct size after ischemia/reperfusion. J Pharniacol Exp Ther2002;303: 1 007-13 
${ }^{20}$-Pietila K, Harnıoinen A, Poyhonen L, et al. Intravenous streptokinase treatment and serum C-reactive protein in patients wiıh acute nıyocardial infarction. Br Hearı J 1987;58:225-9

21_ Pietila K, Harmoinen A, Hermens W, et al. Serum C-reactive protein and infarct size in myocardial infarct patients wiıh a closed versus an open infarct-related coronary arıery after thrombolytic therapy. Eur Heart J 1993; 14:915-9

22_ Doganay B., Okutucu S., Cetin M. Et al Association of Serum Copeptin Levels with Patency of InfarctRelated Arteries in Patients with ST-Segment Elevation Myocardial Infarction. Acta Cardiol Sin 2019;35:360 368 DOI: 10.6515/ACS.201907_35(4).20181101A

23_ Jing Li, Ying Zhou, Yaowen Zhang et al Admission homocysteine is an independent predictor of spontaneous reperfusion and early infarct-related artery patency before primary percutaneous coronary intervention in ST-segment elevation myocardial infarction. Li et al. BMC Cardiovascular Disorders (2018) 18:125 https://doi.org/10.1186/s12872-018-0868-3

24_Cinar T. Cagdaş M. Rencüzogulları İ. et al Prognostic Efficacy of C-reactive Protein/Albumin Ratio in ST-Elevation Myocardial Infarction. Scand Cardiovasc J DOI: 10.1080/14017431.2019.1590628. Epub 2019 Apr 3.

25-Karabag Y, Cagdas M, Rencuzogullari I, et al. Relationship between C-reactive protein/albumin ratio and coronary artery disease severity in patients with stable angina pectoris. J. Clin. Lab. Anal. 32(7), e22457 (2018).

26_ Wang W, Ren D, Wang CS et al Prognostic Efficacy of High-Sensitivity C-reactive Protein to Albumin Ratio in Patients With Acute Coronary Syndrome. Biomark Med. 2019 Jul;13(10):811-820. DOI: 10.2217/bmm-2018-0346. Epub 2019 May 30.

27- Karabag Y, Cagdas, M, Rencuzogullari I, et al. Usefulness of the C-reactive protein/albumin ratio for predicting no-reflow in ST-elevation myocardial infarction treated with primary percutaneous coronary intervention. Eur. J. Clin. Invest. 48(6), e12928 (2018).

${ }^{28}$-Sianos G, Papafaklis MI, Serruys PW. Angiographic thrombus burden classification in patients with ST-segment elevation myo-cardial infarction treated with percutaneous coronary interven-tion. J Invasive Cardiol. 2010;22(10):6B-14B.

${ }^{29}$-Singh M, Berger PB, Ting HH, et al. Influence of coronary throm-bus on outcome of percutaneous coronary angioplasty in the cur-rent era (the Mayo Clinic experience). Am J Cardiol. 2001; 88(10):1091-1096. 